\title{
Gaussian Diamond Primitive Relay with Oblivious Processing
}

\author{
Asif Katz, Michael Peleg ${ }^{(1),(2)}$ and Shlomo Shamai ${ }^{(1)}$ \\ Technion-Israel Institute of Technology ${ }^{(1)}$, Rafael $\operatorname{ltd}^{(2)}$
}

\begin{abstract}
We examine the frequency-selective Gaussian primitive diamond relay channel, where the signal received at both relays is described by identical frequency-selective Gaussian channels, and where the relays are connected to the destination via noiseless fronthaul links of a given capacity. The frequency response of the filters are available to all system components. We characterize analytically the Gaussian input frequency power distribution that maximizes the optimal information rate of the oblivious (code independent) relay operation achieved by joint decompression-decoding (or optimized Wyner-Ziv) procedure.

Index Terms - Oblivious processing, Gaussian information bottleneck, quantization, finite link capacity, relay, waterpouring.
\end{abstract}

\section{INTRODUCTION}

Relaying is used in order to improve the performance of a communication system by using intermediate nodes. An example is 'cloud' communication [1] and Cloud Radio Access Network (CRAN) [2], where there are several base stations, each has error free, fronthaul link to a cloud computing central processor. By joint processing the signals of the different base stations, we can increase the spectral efficiency of the cellular network. Another example is the Remote Radio Heads (RRH) system connected to base stations with Common Public Radio Interface (CPRI) [3]. The fronthaul importance is shown in [4]. One interesting model of the relay system is the oblivious relay. In this model there is no a priori knowledge of the modulation or the coding at the relay, thus the relaying system is universal and can serve many diverse users and operators, CPRI is in this category. This work present methods designed to reduce the bitrates from the relays to the final destination.

A single relay scheme and the optimal frequencydependent allocation of power and bitrate was investigated in [5], [6] using Gaussian bottleneck results [7] and Shannon's incremental frequency approach [8]. The problem can be compared to the remote source coding, only with logarithmic loss instead of mean square error in [9] The scheme for this relay is shown in [5, Fig. 2]. Frequency-flat channels were shown in [10]. In [5] the flat channel filter H(f) results were found, along with frequency dependent channel filter response. The expressions rely on standard coding theorems [11]. The optimization algorithms for the quantizers and their performance are shown in [12].

In this paper we expand the system to a model with two relay channels, as shown in Fig. 1. The problem of parallel independent Gaussian channels with cross frequency dependent interference, was shown in [13]. We solve analytically the identical frequency-selective Gaussian diamond relay channel, and determine the optimal input spectrum under a fixed power constraint, the special case of which is the classical water-pouring result (infinite fronthaul link capacities). The analytical calculation is done by symbolic calculation using MATLAB Symbolic Toolbox.

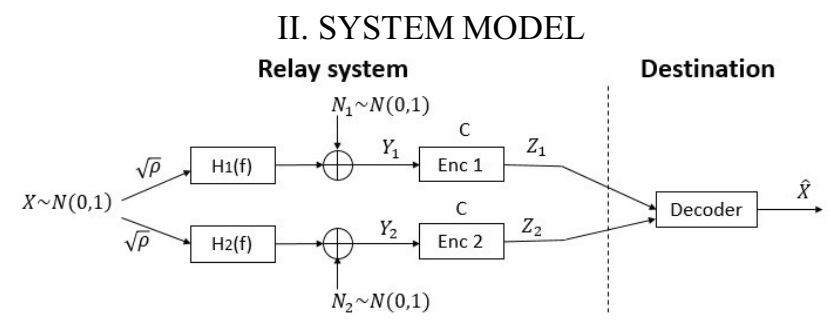

Fig. 1. Two relay channels communication system

The system model is a real Gaussian signal $\mathrm{X}$ over two AWGN relay channels. Each relay channel is with signal to noise ratio (SNR) equal to $\rho$, has frequency dependent channel response and rate limited encoder. In this paper we limit the model for the case where $\mathrm{H}_{1}(\mathrm{f})=\mathrm{H}_{2}(\mathrm{f})$. Each relay channel has limited bit rate $C$ [bits/ channel use] from the encoder to the decoder at the destination. The relay encoders are oblivious of the code used, and they do not communicate with each other. We aim to maximize the mutual information between $\mathrm{X}$ and $Z \triangleq\left(Z_{1}, Z_{2}\right)$ subject to the rate constraint between the relays and the destination.

\section{PRELIMINARIES}

In this section we discuss preliminaries about the bottleneck problem, waterpouring method and relevant results from [5].

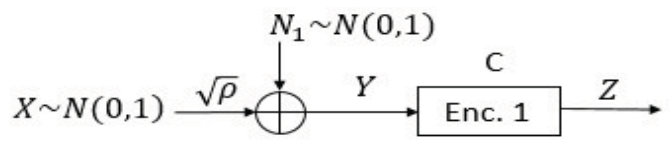

Fig. 2. One relay channel communication system

\section{A. Bottleneck}

The optimal performance of the oblivious system with no interference, is governed by the Gaussian Information Bottleneck (GIB) [14] and was analyzed in [7], [15], [16]. While different than minimum mean squared distortion based remote source coding [17], [18], [19] it is the remote source coding with logarithmic distortion [20]. The system rate of the one relay channel system, shown in Fig. 2, is given by solving the problem:

$$
\begin{aligned}
& I(C)=\max _{P(z \mid y)} I(X ; Z) \\
& \text { s.t } I(Y ; Z) \leq C
\end{aligned}
$$

The solution for that optimization problem on a specific frequency, which is presented in [15], is solved using 
bottleneck results and standard Euler-Lagrange [21]. The expression is:

$$
I(\rho, C)=\frac{1}{2} \log _{e}\left(\frac{1+\rho}{1+\rho e^{-2 C}}\right)
$$

This is the optimal mutual information between $\mathrm{X}$ and $\mathrm{Z}$ subject to the constraint on the rate between $\mathrm{Y}$ and $\mathrm{Z}$.

\section{B. Water-Pouring}

In this method, that was shown in [5] and [6], we split the channel into separate bands, each with bandwidth of $d f$. In our Gaussian model, the different bands are independent. For each band we assign rate of $0.5 C(f)$ per channel use. Then rate in the band equals $C(f) d f$, because we have $2 d f$ channel uses for each band (Nyquist). The SNR of each band is dependent on the spectrum allocation $S(f)$ of the input signal $\mathrm{X}$, equals $S(f)|H(f)|^{2}$. Therefore, the frequency dependent rate derived from (2) is:

$$
I(f, S(f), C(f))=\log _{e}\left(\frac{1+S(f)|H(f)|^{2}}{1+S(f)|H(f)|^{2} e^{-C(f)}}\right)
$$

The optimization problem in (1) becomes:

$$
\begin{aligned}
& \max _{S(f), C(f)} \int_{0}^{W} I(f, S(f), C(f)) d f \\
& \text { s.t. } \int_{0}^{W} S(f) d f \leq P, \int_{0}^{W} C(f) d f \leq C
\end{aligned}
$$

The solution for this problem is shown in [5], using the Lagrange multipliers method. We next expand this to the case of two relay channels. We will also show upper and lower bounds where the encoders are either cooperative or independent.

\section{SYSTEM DESIGN AND ANALYSIS}

Now we investigate the two channels relay system shown in Fig. 1. The transmitter uses classic codes, resulting in Gaussian-distributed $\mathrm{X}$. The compression in each relay is done by using single channel remote source-coding with Wyner-Ziv compression as in $[1,19,21]$.

At a single frequency, the optimization problem we solve for the system in Fig. 1, special case of [20, Theorem 5]:

$I(C)$

$=\max _{r_{1}, r_{2} \geq 0}\left\{\min _{S \in\{1,2\}}\left\{\frac{1}{2} \log _{2}\left(1+\rho \sum_{t \in S^{C}}\left(1-2^{-2 r_{t}}\right)+\sum_{t \in S}\left[C-r_{t}\right]\right)\right\}\right\}$

The set $\mathrm{S}$ is the set of all the relays, in our case of size 2 , and $r_{t}$ indicate the bandwidth wasted by quantizing the additive noise for $\mathrm{t}=1,2$. Solution for this problem was shown in [20] and [22] for the case of CRAN with no time sharing. We are interested in the solution of the real signal case, shown in [20, Corollary 5].

Using our notation, the required expression is:

$$
\begin{aligned}
& I(\rho, C)= \\
& \frac{1}{2} \log _{2}\left(1+2 \cdot \rho \cdot 2^{-4 C} \cdot\left(2^{4 C}+\rho-\sqrt{\rho^{2}+(1+2 \cdot \rho) \cdot 2^{4 C}}\right)\right)
\end{aligned}
$$

This result is achieved either with joint decompressiondecoding or optimized (time sharing) Wyner-Ziv approach (see $[22,22,23]$, for the general $\mathrm{K}$ relays model, accounting for joint decompression/decoding). First we investigate the expression above and show that:

$$
\begin{gathered}
I(\rho, C) \stackrel{C \rightarrow \infty}{\longrightarrow} \frac{1}{2} \log _{2}(1+2 \cdot \rho) \\
I(\rho, C) \stackrel{\rho \rightarrow \infty}{\longrightarrow} 2 C
\end{gathered}
$$

Proof is in Appendix - A. This behavior for different $\mathrm{C}$ rates is shown on Fig. 3.

Now we use the same water-pouring approach shown in [5] and (3), now with (6), yielding (9).

$$
\begin{aligned}
& I(f, S(f), C(f))= \\
& \log _{2}\left(1+2 \cdot B(f) \cdot 2^{-2 C(f)} \cdot\left(2^{2 C(f)}+B(f)-\sqrt{A(f)}\right)\right) \\
& A(f) \triangleq(B(f))^{2}+(1+2 \cdot B(f)) \cdot 2^{2 C(f)} \\
& B(f) \triangleq S(f)|H(f)|^{2}
\end{aligned}
$$

The problem we now solve is:

$$
\begin{aligned}
& \max _{S(f), C(f)} \int_{0}^{W} I(f, S(f), C(f)) d f \\
& \text { s.t. } \int_{0}^{W} S(f) d f \leq P, \int_{0}^{W} C(f) d f \leq C
\end{aligned}
$$

Using the Lagrange multipliers method as shown in [5], we get the following problem:

$$
L\left(f, \hat{S}, \hat{C}, \lambda_{c}, \lambda_{s}\right)=I(f, \hat{S}, \hat{C})-\lambda_{s} \cdot \hat{S}-\lambda_{c} \cdot \hat{C}
$$

Where $\hat{S} \triangleq S(f), \hat{C} \triangleq C(f)$. We also 7denote $H(f) \triangleq h$. And now in order to solve the optimization problem, we search for the optimal solution point where the gradient equals zero. Therefore we need to solve the following equations:

$$
\nabla L=\left(\frac{d L}{d S}, \frac{d L}{d C}\right)=(0,0)
$$

As shown in [5], the optimal solution of $\mathrm{C}$ and $\mathrm{S}$ 6at each frequency is either in the concave region of (6) or zero. This will ensure that there is only one non-zero solution for the optimization problem. The concavity is determined by the hessian matrix:

$$
H=\left(\begin{array}{cc}
f_{x x} & f_{x y} \\
f_{y x} & f_{y y}
\end{array}\right)
$$

Where for our function $f_{x y}=f_{y x}$. In the concave region the hessian matrix terms fulfill the following inequalities:

$$
\begin{aligned}
& f_{x x} \leq 0 \\
& f_{x x} \cdot f_{y y}-f_{x y}{ }^{2} \geq 0
\end{aligned}
$$

In order to find the concave region we used numerical calculation with symbolic tools in MATLAB. Then we found the region where $I(f, \hat{S}, \hat{C})$ of (9), fulfills (14). The region is shown in Fig. 4 . It can be seen from Fig. 4 that the minimum 
rate which defines the border line between the concave and non-concave region is $\mathrm{C}=0.5$. The region curve is similar to the region shown in [5].

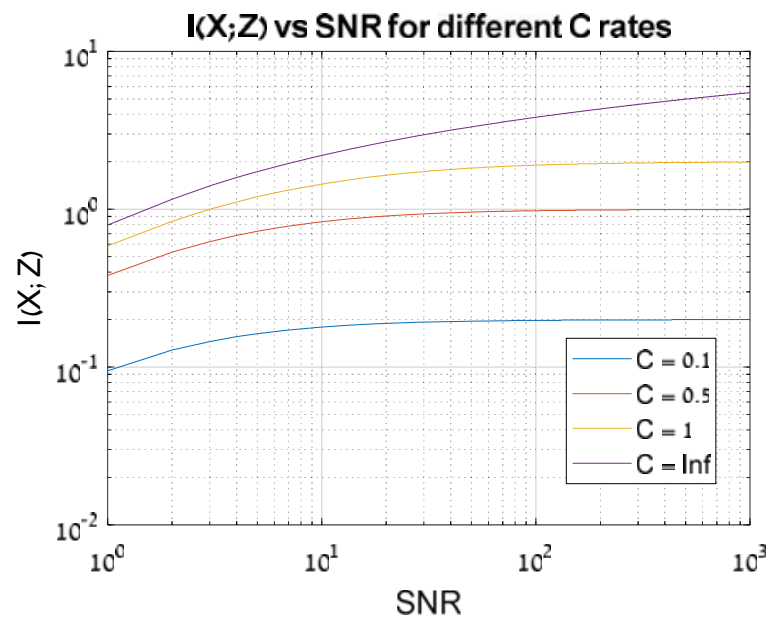

Fig. 3. Two relay channels communication system - performance at a single frequency.

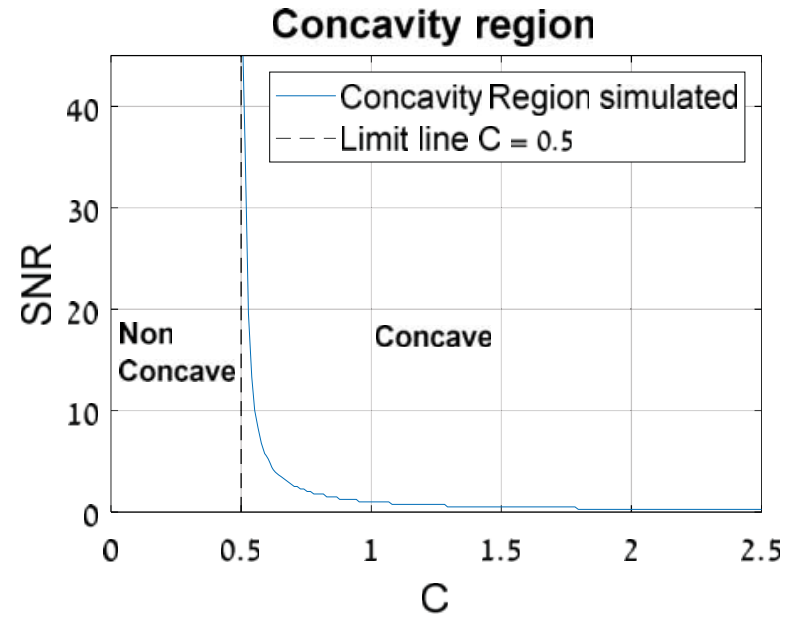

Fig. 4. Concavity region of $I(f, \hat{S}, \hat{C})$.

(9), (11), (12) gives the following equation system:

$$
\left\{\begin{array}{l}
\lambda_{s}=\frac{d I}{d S}=\frac{2 h^{2}}{2^{2 c}} \cdot \frac{\left(h^{2} s+2^{2 c}-X\right)-s \cdot\left(\frac{h^{4} s+h^{2} 2^{2 c}}{X}-h^{2}\right)}{\log _{e} 2\left(\frac{2}{2^{2 c}} \cdot h^{2} s \cdot\left(h^{2} s+2^{2 c}-X\right)+1\right)} \\
\lambda_{c}=\frac{d I}{d C}=\frac{2 h^{2} s}{X}
\end{array}\right.
$$

$$
X \triangleq \sqrt{2^{2 c} \cdot\left(2 \cdot h^{2} s+1\right)+h^{4} s^{2}}
$$

Solving (15) for $\mathrm{S}$ and $\mathrm{C}$ results in two different solutions for the power and rate frequency allocation, shown in (17). We use the same method described in [5] in order to find the optimal solution using grid search on the Lagrange multipliers. For each grid point we do the following:

1) Find the frequency allocation functions of solution 1 from (17).

2) Calculate the total power and rate over the frequency domain.
3) If we do not fulfill the power or rate constraints of (10), we sort the frequencies by their information values derived by (9).

4) Then we exclude the frequencies with the least values until the power and rate constraints are fulfilled, this is done by assigning zero power and zero rate to those frequencies.

5) Next we calculate the total information over the frequency domain, as in (10).

Finally we choose the grid point with the maximal value of the total information.

\section{A. Frequency flat filter response}

First we investigate the solution for the case where $H(f) \triangleq h \equiv 1$. In Appendix - B we derive the Lagrange coefficients region, using (15), which for this case is:

$$
\begin{aligned}
& 0 \leq \lambda_{c} \leq 2 \\
& 0 \leq \lambda_{s} \leq \frac{2}{\log _{e} 2}
\end{aligned}
$$

Using (16), we can perform grid search on the Lagrange coefficients region, as was done in [5], in order to find the optimal solution. Solving (15) yielded the following two solutions:

$$
\begin{aligned}
& \mathrm{s}_{i}=-\frac{h^{2} \lambda_{c}-2 h^{2}+\lambda_{s} \log _{e} 2+\psi_{i} \cdot \sqrt{J}}{4 h^{2} \lambda_{s} \log _{e} 2} \\
& J=h^{4} \lambda_{c}{ }^{2}-6 h^{2} \lambda_{c} \lambda_{s} \log _{e} 2-4 h^{4} \lambda_{c} \\
& +\lambda_{s}{ }^{2}\left(\log _{e} 2\right)^{2}-4 h^{2} \lambda_{s} \log _{e} 2+4 h^{4} \\
& c_{i}=\frac{1}{2 \log _{e} 2} \log \left(-\frac{h^{4} s_{i}{ }^{2}\left(\lambda_{c}{ }^{2}-4\right)}{\lambda_{c}{ }^{2} \cdot\left(2 \cdot s_{i} \cdot h^{2}+1\right)}\right) \\
& \psi_{i}= \begin{cases}-1, i=1 \\
1, i=2\end{cases} \\
& i \in\{1,2\}
\end{aligned}
$$

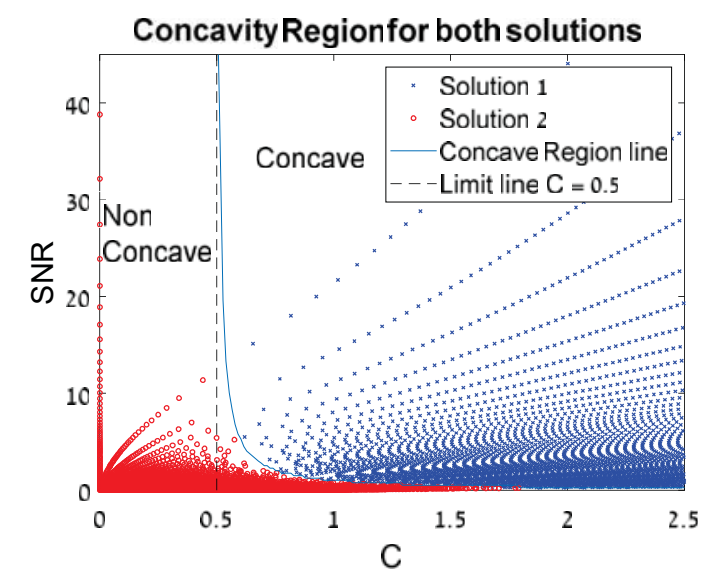

Fig. 5. Solutions 1 and 2 over the SNR-C plane with concave region border line.

The square root in the solution in (17) also restricts the grid area to a region where the solution is real. Complex values of the solutions over the grid were discarded in the numerical analysis. Checking both solutions over grid of the Lagrange coefficients, substitute the SNR and C rate from (17), then check the hessian concave condition in (14). The solutions 
points over the grid are shown in Fig. 5. We denote solution number 1 or 2 as $\mathrm{i}=1$ or $\mathrm{i}=2$ of (17).

It can be seen from Fig. 5 that the solution which is inside the concave region is solution 1 . The other solution is always outside the concave region. Thus solution 1 is our optimization problem solution. For this solution we can now find the optimal solution for a specific example shown in [5], for: $\mathrm{P}=100[\mathrm{Watt}], \mathrm{C}=9[$ nats/sec $]$ and $\mathrm{W}=10[\mathrm{~Hz}]$. The frequency allocation of the optimal solution is shown in Fig. 6(a). Interesting behavior of the optimal solution, shown also in [5], is that there are frequencies with zero allocation.

(a)
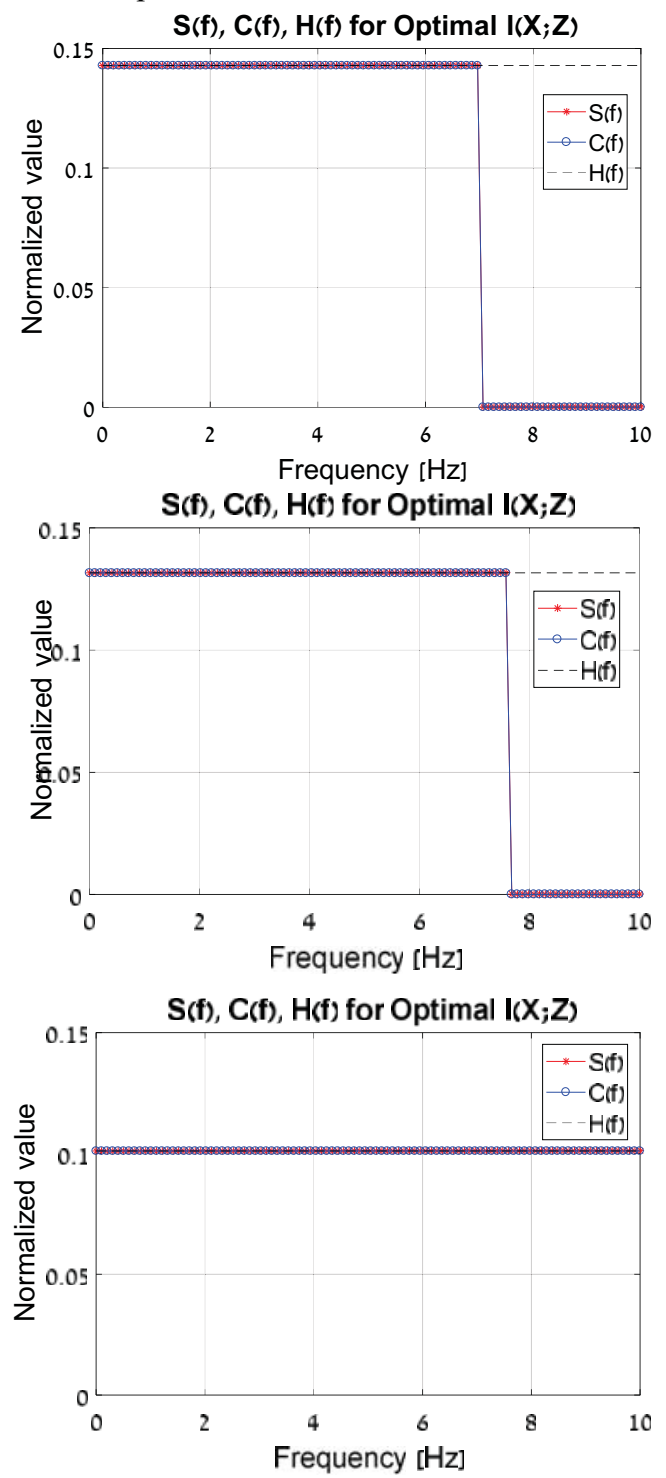

Fig. 6. Frequency allocation of the optimal solution with a constant $H(f)$ for $P=100$ [Watt], $C=9[$ nats/sec] and $W=10[\mathrm{~Hz}]$. (a) our scheme. (b) upper bound. (c) lower bound.

\section{B. Frequency dependent filter response}

Next we show the case of frequency dependent filter. We use the same filter as in [5]. The filter is: $H(f)=\max _{f}\left|H_{A}(f)\right|-H_{A}(f)$ where

$H_{A}(f)=\alpha_{1} \cdot G_{N\left(f_{1}, 1\right)}(f)+\alpha_{2} \cdot G_{N\left(f_{2}, 1\right)}(f)$ and $G_{N\left(f_{1}, 1\right)}(f)$ is the Gaussian curve with mean $f_{1}$ and variance 1 . The results are shown in Fig. 7. Clearly the allocation of the power and bitrate resources is a monotonically increasing function of the channel response $\mathrm{H}(\mathrm{f})$ and no resources at all are allocated to regions in which $\mathrm{H}(\mathrm{f})$ is too weak.

\section{Upper and lower bounds}

We compare our results to upper and lower bounds. The upper bound is the case of cooperative encoding of Enc 1 and Enc 2 in Fig. 1. In this case, the encoders can share information and operate jointly and the problem is similar to the scheme in Fig. 2, the two encoders system equals one encoder system with vectors input $(\mathrm{Y} 1, \mathrm{Y} 2)$ and output $(\mathrm{Z1}, \mathrm{Z} 2)$. The SNR is doubled because the signals of both channels are identical and the noises are independent. Also the total compression rate of the system is doubled, because we have two rates of both encoders. We can use the formula of (3) for this case:

$I(f, S(f), C(f))=\log _{2}\left(\frac{1+2 S(f)|H(f)|^{2}}{1+2 S(f)|H(f)|^{2} 2^{-2 C(f)}}\right)$

(a)
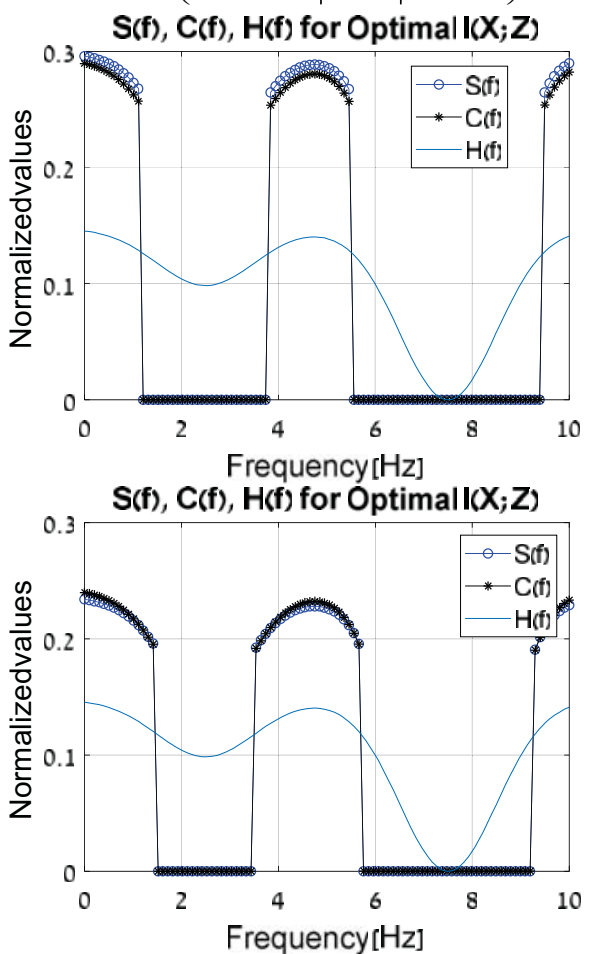

(b)

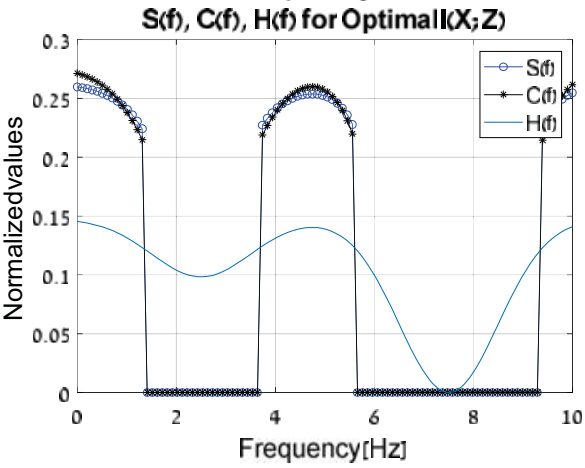

Fig. 7. Frequency allocation of the optimal solution with a frequency dependent $H(f)$ for $P=100[$ Watt], $C=9$ [nats/sec] and $W=10[\mathrm{~Hz}]$. (a) our scheme. (b) upper bound .(c) lower bound. 
TABLE I

SUMMARY OF I(X;Z) VALUES

\begin{tabular}{|l|c|c|c|c|}
\hline & \multicolumn{4}{|c|}{$(\mathrm{P}=100[$ Watt], W=10[Hz], C=9[nats/sec] unless specified $)$} \\
Case & $\begin{array}{c}\text { Our optimal } \\
\text { scheme }\end{array}$ & $\begin{array}{c}\text { Collaborative } \\
\text { encoding - } \\
\text { upper bound }\end{array}$ & $\begin{array}{c}\text { Independently encoding } \\
\text { - lower bound }\end{array}$ & $\begin{array}{c}\mathrm{I}(\mathrm{X} ; \mathrm{Z}) \\
\mathrm{C}=\infty\end{array}$ \\
\hline Frequency flat filter & 15.31 & 16.44 & 12.98 & 44.21 \\
\hline $\begin{array}{l}\text { Frequency } \\
\text { dependent filter }\end{array}$ & 6.8 & 8.16 & 6.54 & 10.48 \\
\hline
\end{tabular}

The lower bound is a system in which each relay operates as in [5], independently and without the Wyner-Ziv compression and the destination just averages the two signals, thus improving the final SNR by a factor of 2 . To evaluate it we use (3), convert the information rate to the SNR of a single decompressed signalby the AWGN capacity formula, multiply by a factor of 2 and convert back to bitrate. The lower bound expression is:

$$
\begin{aligned}
& I(f, S(f), C(f)) \\
& =\log _{2}\left(\frac{1+2 S(f)|H(f)|^{2}-S(f)|H(f)|^{2} 2^{-C(f)}}{1+2 S(f)|H(f)|^{2} 2^{-C(f)}}\right)
\end{aligned}
$$

\section{CONCLUSIONS}

We investigated and derived the optimal frequencydependent allocation of power and bitrate for a pair of oblivious relays operating over the AWGN frequencydependent channel and using classical Gaussian codes. This is a basic extension of the single relay system [5]. We investigated the loss incurred by lack of cooperation between the relays which would necessitate an additional communication link between them. As shown in Table 1, the advantage of applying the distributed compression (optimized Wyner-Ziv) technique is evident and the limit on relay to destination bitrates and the lack of communication between the relays limit the performance. As in [5], the optimal allocation, shown in Fig. 6, 7, is zero for some frequencies even over some frequency-flat channels due to the need to concentrate power and bitrate resources.

\section{ACKNOWLEDGMENT}

This work has been supported by the European Union's Horizon 2020 Research And Innovation Programme, grant agreement no. 694630 .

\section{APPENDIX}

In this section, due to space limitations, we shortly elaborate on the proofs of the above results.

\section{A. Information rate limits}

We develop the limits of (6). First we develop the limit for $C \rightarrow \infty$ :

$I(\rho, C)=0.5 \cdot \log _{2}\left(1+2 \cdot \rho \cdot 2^{-4 C} \cdot\left(2^{4 C}+\rho-\sqrt{\rho^{2}+(1+2 \cdot \rho) \cdot 2^{4 C}}\right)\right)$

$=0.5 \cdot \log _{2}\left(1+2 \cdot \rho \cdot\left(1+\rho 2^{-4 C}-2^{-4 C} \sqrt{\rho^{2}+(1+2 \cdot \rho) \cdot 2^{4 C}}\right)\right)$

$=0.5 \cdot \log _{2}\left(1+2 \cdot \rho \cdot(1+\underbrace{\rho 2^{-4 C}}_{c \rightarrow 0}-\underbrace{\underbrace{2^{-2 C}}_{C \rightarrow \infty} \sqrt{\underbrace{\rho^{2} 2^{-4 C}}_{c \rightarrow 0}+(1+2 \cdot \rho)}}_{\underbrace{}_{C \rightarrow \infty} \rightarrow 0})\right)$

$\stackrel{C \rightarrow \infty}{\longrightarrow} 0.5 \cdot \log _{2}(1+2 \cdot \rho)$

$I(\rho, C)$

Next we develop the limit for $\rho \rightarrow \infty$ :

$=0.5 \cdot \log _{2}\left(1+2 \cdot \rho^{2} \cdot 2^{-4 C} \cdot\left(\frac{2^{4 C}}{\rho}+1-\sqrt{1+\frac{(1+2 \cdot \rho) \cdot 2^{4 C}}{\rho^{2}}}\right)\right)$

$\approx 0.5 \cdot \log _{2}\left(1+2 \cdot \rho^{2} \cdot 2^{-4 C} \cdot\left(\frac{2^{4 C}}{\rho}+1-\left(1+\frac{1}{2} \frac{(1+2 \cdot \rho) \cdot 2^{4 C}}{\rho^{2}}-\frac{1}{8}\left(\frac{(1+2 \cdot \rho) \cdot 2^{4 C}}{\rho^{2}}\right)^{2}\right)\right)\right.$

Where we used the Taylor series near $\mathrm{x}=0$ :

$$
\sqrt{1+x} \approx 1+\frac{1}{2} x-\frac{1}{8} x^{2}
$$

Continue with the expression inside the logarithm:

$1+2 \cdot \rho^{2} \cdot 2^{-4 C} \cdot\left(\frac{2^{4 C}}{\rho}-\frac{1}{2} \frac{(1+2 \cdot \rho) \cdot 2^{4 C}}{\rho^{2}}+\frac{1}{8}\left(\frac{(1+2 \cdot \rho) \cdot 2^{4 C}}{\rho^{2}}\right)^{2}\right)$

$=1+2 \cdot \rho^{2} \cdot 2^{-4 C} \cdot\left(\frac{2^{4 C}}{\rho}-\frac{2^{4 C}}{2 \rho^{2}}-\frac{2^{4 C}}{\rho}+\frac{1}{8}\left(\frac{(1+2 \cdot \rho) \cdot 2^{4 C}}{\rho^{2}}\right)^{2}\right)$

$=1+2 \cdot \rho^{2} \cdot 2^{-4 C} \cdot\left(-\frac{2^{4 C}}{2 \rho^{2}}+\frac{1}{8}\left(\frac{(1+2 \cdot \rho) \cdot 2^{4 C}}{\rho^{2}}\right)^{2}\right)$

Now back to the information expression we get:

$I(\rho, C)=0.5 \cdot \log _{2}\left(2^{4 C}\left(1+\frac{1}{\rho}+\frac{1}{4 \rho^{2}}\right)\right)$

$\stackrel{\rho \rightarrow \infty}{\longrightarrow} 0.5 \cdot \log _{2}\left(2^{4 C}\right)=2 C \log _{2}(2)=2 \mathrm{C}$ 


\section{B. Lagrange coefficients region}

We derive here the region of the Lagrange coefficients from (15). First we denote that $s, c, X \geq 0$. Thus $\lambda_{c} \geq 0$. For the upper bound we use (14) condition:

$$
\frac{d \lambda_{c}}{d C}=\frac{d^{2} I}{d C^{2}} \leq 0
$$

This means that the maximum value is achieved at $\mathrm{C}=0$.

$$
\begin{aligned}
& \lambda_{c} \leq \max _{C} \frac{2 h^{2} s}{X}=\frac{2 h^{2} s}{\sqrt{2 \cdot h^{2} s+1+h^{4} s^{2}}} \\
& =\frac{2 h^{2} s}{\sqrt{\left(h^{2} s+1\right)^{2}}}=2 \frac{h^{2} s}{h^{2} s+1} \leq 2
\end{aligned}
$$

Now develop $\lambda_{S}$ :

$$
\begin{aligned}
& \lambda_{s}=\frac{d I}{d S}=\frac{2 h^{2}}{2^{2 c}} \cdot \frac{\left(h^{2} s+2^{2 c}-X\right)-s \cdot\left(\frac{h^{4} s+h^{2} 2^{2 c}}{X}-h^{2}\right)}{\log _{e} 2\left(\frac{2}{2^{2 c}} \cdot h^{2} s \cdot\left(h^{2} s+2^{2 c}-X\right)+1\right)} \\
& =\frac{2 h^{2}}{2^{2 c}} \cdot \frac{X\left(\frac{h^{2} s+2^{2 c}}{X}-1\right)-h^{2} s \cdot\left(\frac{h^{2} s+2^{2 c}}{X}-1\right)}{\log _{e} 2\left(\frac{2}{2^{2 c}} \cdot h^{2} s \cdot\left(h^{2} s+2^{2 c}-X\right)+1\right)} \\
& =\frac{2 h^{2}}{2^{2 c}} \cdot \frac{\frac{1}{X}\left(h^{2} s+2^{2 c}-X\right)\left(X-h^{2} s\right)}{\log _{e} 2\left(\frac{2}{2^{2 c}} \cdot h^{2} s \cdot\left(h^{2} s+2^{2 c}-X\right)+1\right)}
\end{aligned}
$$

We check each expression in the above equation.

1)

$$
\begin{aligned}
& X-h^{2} s=\sqrt{\underbrace{2^{2 c} \cdot\left(2 \cdot h^{2} s+1\right)}_{\geq 0}+h^{4} s^{2}}-h^{2} s \geq \sqrt{h^{4} s^{2}}-h^{2} s=0 \\
& \text { 2) } \\
& h^{2} s+2^{2 c}-X=h^{2} s+2^{2 c}-\sqrt{2^{2 c} \cdot\left(2 \cdot h^{2} s+1\right)+h^{4} s^{2}} \\
& =h^{2} s+2^{2 c}-\sqrt{\left(h^{2} s+2^{2 c}\right)^{2}+2^{2 c} \underbrace{\left(1-2^{2 c}\right)}_{\leq 0}} \\
& \geq h^{2} s+2^{2 c}-\sqrt{\left(h^{2} s+2^{2 c}\right)^{2}}=0
\end{aligned}
$$

From the above we can infer that: $\lambda_{S} \geq 0$.

Now again:

$$
\begin{aligned}
& \frac{d \lambda_{s}}{d S}=\frac{d^{2} I}{d S^{2}} \leq 0 \\
& \lambda_{s}=\max _{s} \frac{2 h^{2}}{2^{2 c}} \cdot \frac{\left(h^{2} s+2^{2 c}-X\right)-s \cdot\left(\frac{h^{4} s+h^{2} 2^{2 c}}{X}-h^{2}\right)}{\log _{e} 2\left(\frac{2}{2^{2 c}} \cdot h^{2} s \cdot\left(h^{2} s+2^{2 c}-X\right)+1\right)}
\end{aligned}
$$

So:

$$
=\frac{2 h^{2}}{2^{2 c}} \cdot \frac{\left(2^{2 c}-2^{c}\right)}{\log _{e} 2}=2 h^{2} \cdot \frac{\left(1-2^{-c}\right)}{\log _{e} 2} \leq \frac{2 h^{2}}{\log _{e} 2}
$$

\section{REFERENCES}

[1] A. Sanderovich, S. Shamai, and Y. Steinberg, "Distributed mimo receiver-achievable rates and upper bounds," IEEE Trans Information Theory, vol. 55, no. 10, pp. 4419-4438, October 2009.

[2] S.H. Park, O. Simeone, O. Sahin, and S. Shamai, "Fronthaul compression for cloud radio access networks: Signal processing advances inspired by network information theory," IEEE Signal Processing Magazine, vol. 31, no. 6, pp. 69-79, November 2014.

[3] A. de la Oliva, J. A. Hern'andez, D. Larrabeiti, and A. Azcorra, "An overview of the cpri specification and its application to c-ran-based lte scenarios," IEEE Communications Magazine, vol. 54, no. 2, pp. 152-159, February 2016.

[4] D. Camps-Mur et al., "5G-XHaul: A Novel Wireless-Optical SDN Transport Network to Support Joint 5G Backhaul and Fronthaul Services," in IEEE Communications Magazine, vol. 57, no. 7, pp. 99-105, July 2019.

[5] A. Homri, M. Peleg, and S. Shamai (Shitz), "Oblivious processing in a fronthaul constrained gaussian channel", Proc. IEEE International Conference on the Science of Electrical Engineering (ICSEE), pp. 1-5, November 2016.

[6] A. Homri, M. Peleg, and S. Shamai, "Oblivious FronthaulConstrained Relay for a Gaussian Channel", IEEE Transactions on communications, vol. 66, no. 11, November 2018.

[7] G. Chechik, A. Globerson, N. Tishby, and Y. Weiss, "Information bottleneck for gaussian variables", Journal of Machine Learning Research, vol. 6, pp. 165-188, January 2005.

[8] C. Shannon, "Communication in the presence of noise", Proc. IRE, vol. 37, no. 1, pp. 10-21, January 1949.

[9] A. Kipnis, Y. C. Eldar, and A. J. Goldsmith, "Analog-to-digital compression: A new paradigm for converting signals to bits", IEEE Signal Processing Magazine, vol. 35, no. 3, pp. 16-39, May 2018.

[10] R. Siddharth, M. Medard, and L. Zheng, "Fiber aided wireless network architecture", IEEE Journal on selected areas in communications, vol. 29, no. 6, pp. 1284-1294, June 2011.

[11] R. G. Gallager, Information Theory and Reliable Communications. New York: J. Wiley, 1968.

[12] S. Hassanpour, D. Wuebben, and A. Dekorsy, "Overview and investigation of algorithms for the information bottleneck method", 11th International ITG Conference on Systems, Communications and Coding, February 2017.

[13] L. Brandenburg and A.Wyner, "Capacity of the gaussian channel with memory: The multivariate case", Bell System Technical Journal, vol. 53, pp. 745-779, May 1974.

[14] N. Tishbi, F. Pereira, and W. Bialek, "Information bottleneck method," Proc. 37th Allerton Conference on Communication, Control and Computing, pp. 368-377, September 1999.

[15] A. Winkelbauer and G. Matz, "Rate-information-optimal gaussian channel output compression," 48th Annual Conference on Information Sciences and Systems (CISS), no. 1-5, August 2014.

[16] A.Winkelbauer, S. Farthofer, and G. Matz, "The rateinformation trade-off for gaussian vector channels," IEEE International Symposium on Information Theory (ISIT), pp. 2849 - 2853, July 2014.

[17] R. Dobrushin and B. Tsybakov, "Information transmission with additional noise", IRE Transactions on Information Theory, vol. 8, no. 5, pp. 293-304, September 1962.

[18] C. Tian and J. Chen, "Remote vector gaussian source coding with decoder side information under mutual information and distortion constraints," IEEE Transactions on Information Theory, vol. 55, no. 10, pp. 4676-4680, October 2009.

[19] A. Kipnis, A. Goldsmith, Y. Eldar, and T. Weissman, "Distortion-rate function of sub-nyquist sampled gaussian sources", IEEE Transactions on Information Theory, vol. 62, pp. 401-429, January 2016.

[20] A. Sanderovich, S. Shamai (Shitz), Y. Steinberg and G. Kramer, "Communication via decentralized processing", IEEE Trans. Information Theory, vol. 54, no. 7, pp. 3008-3023, July 2008.

[22] I. E. Aguerri, A. Zaidi, G. Caire and S. Shamai (Shitz), "On the Capacity of Cloud Radio Access Networks with Oblivious Relaying", to appear IEEE Transactions on Information Theory (arXiv:1710.09275).

[23] A. Zaidi and S. Shamai (Shitz), "Perspectives on Information Bottleneck Problems", Information Theory \& Applications (ITA2019), February 2019.

[24] I. Estella and A. Zaidi, "Distributed Information Bottleneck Method for Discrete and Gaussian Sources", International Zurich Seminar on Information and Communication, February 2018. 